\title{
Seizure-Associated, Aberrant Neurogenesis in Adult Rats Characterized with Retrovirus-Mediated Cell Labeling
}

\author{
Sebastian Jessberger, ${ }^{\star}$ Chunmei Zhao, ${ }^{\star}$ Nicolas Toni, Gregory D. Clemenson Jr, Yan Li, and Fred H. Gage \\ Laboratory of Genetics, The Salk Institute for Biological Studies, La Jolla, California 92037
}

Seizure activity within the hippocampal circuitry not only affects pre-existing structures, but also dramatically increases the number of newborn granule cells. A retroviral strategy was used to label dividing cells and their progeny in the adult dentate gyrus and to analyze the impact of epileptic activity on adult-generated cells labeled before or after seizures. We show that epileptic activity led to dramatic changes in the neuronal polarity, migration, and integration pattern of newborn granule cells, depending on the time of birth in relation to the epileptic insult. Aberrant neurons were stably integrated into the dentate circuitry, and the consequences on hippocampal neurogenesis were long lasting. The data presented characterized the consequences of seizure-associated plasticity on adult neurogenesis leading to long-term structural changes in the hippocampal circuitry that might represent a pivotal component of the epileptic disease process.

Key words: neurogenesis; hippocampus; seizure; basal dendrite; dendritic spine; maturation; epilepsy

\section{Introduction}

Mesial temporal lobe epilepsy (mTLE) affects multiple levels of human behavior and frequently results in severe disabilities (Helmstaedter et al., 2003; Elger et al., 2004; von Lehe et al., 2006). Excess neuronal activity in the course of the epileptic disease process not only impacts pre-existing structures, but also exerts influence on endogenous neural stem cells in the adult brain. Previous studies have shown that seizure activity in rodent models of mTLE dramatically increases the proliferation of neurogenic progenitors in the subventricular zone of the lateral ventricle and the hippocampal dentate gyrus, the two neurogenic areas of the adult brain (Bengzon et al., 1997; Parent et al., 1997, 2002). Special attention has been paid to hippocampal neurogenesis after seizures because mTLE commonly leads to an impairment in the hippocampus-dependent processes underlying certain forms of learning and memory (Stafstrom et al., 1993; Helmstaedter, 2002; Stefan and Pauli, 2002). Conventional analyses of seizure-induced neurogenesis have used bromodeoxyuridine (BrdU) labeling of newborn cells or endogenous/transgenic markers that are transiently expressed in newborn neurons. These studies suggested that seizure activity accelerated the early stages of neuronal maturation and that a fraction of hippocampal granule cells generated in response to seizures displayed aberrant basal dendrites or was ectopically localized within the hilar/CA3 border (Parent et al., 1997; Scharfman et al., 2000; Overstreet-

\footnotetext{
Received Jan. 10, 2007; revised July 12, 2007; accepted July 16, 2007.

This work was supported by the Deutsche Forschungsgemeinschaft (Je297/1-1 to S.J.), the American Epilepsy Society (S.J., C.Z.), and grants from the National Institute on Aging, National Institute of Neurological Disorders and Stroke, and the Lookout Fund (F.H.G.). We thank Mary Lynn Gage for editing this manuscript.

*S.J. and C.Z. contributed equally to this work.

Correspondence should be addressed to Fred H. Gage, Laboratory of Genetics, The Salk Institute for Biological

Studies, La Jolla, CA 92037. E-mail: gage@salk.edu.

DOI:10.1523/JNEUROSCI.2002-07.2007

Copyright $\odot 2007$ Society for Neuroscience $\quad 0270-6474 / 07 / 279400-08 \$ 15.00 / 0$
}

Wadiche et al., 2006; Shapiro and Ribak, 2006). Given these findings, it has been hypothesized that seizure-induced neurogenesis either represents an attempt by the adult brain to repair the injured structures or is a pathological part of the epileptic disease process (Parent, 2002; Jakubs et al., 2006). One methodological challenge to understanding the role adult neurogenesis might play in epileptogenesis was to specifically visualize the entire cellular morphology of newborn neurons to determine how long the effects on adult neurogenesis last and whether aberrant neurons born after or before status epilepticus (SE) stably integrate into the dentate circuitry. By labeling dividing progenitors and their progeny in the adult dentate gyrus through retrovirus-mediated expression of green fluorescent protein (GFP), we analyzed the impact of kainic acid (KA)-induced seizures on distinct stages of adult neurogenesis. We found that seizure activity strongly perturbed the dendritic polarization of a granule cell population born after the seizures that nonetheless became stably integrated into the dentate circuitry. In contrast, granule cells that were born before seizures showed mossy fiber sprouting but no aberrant dendritic growth. In summary, seizure activity within the hippocampal circuitry exerts stage-specific influences on newborn granule cells, altering their synaptic integration and, ultimately, their dentate gyrus connectivity. Future studies will have to analyze the role that altered neurogenesis might play in humans with TLE.

\section{Materials and Methods}

Animals. All animal procedures were performed in accordance with the protocols approved by the animal care use committee of the Salk Institute for Biological Studies. All animals were purchased from Harlan (San Diego, CA), were kept under a constant $12 \mathrm{~h}$ light/dark cycle and had access to food ad libitum. To induce seizures, $12 \mathrm{mg} / \mathrm{kg}$ KA (Sigma, St. Louis, MO) in PBS was injected intraperitoneally. Control animals received intraperitoneal injections of an identical volume of PBS. For all experiments, only animals that displayed continuous convulsive stage 5 
seizures were included into the seizure groups (Racine, 1972). Clinical SE subsided 2-3 h after the onset of KA. Unless otherwise stated, animals used were 8-10 weeks old ( $170-200 \mathrm{~g})$, female Fisher-344 rats. Stereotactic injections of $1 \mu \mathrm{l}$ of CAG-GFP retrovirus (see below) were placed into the right dentate gyrus of all experimental animals at the time points indicated below (coordinates from bregma: -2 anteroposterior, -2 mediolateral, -3.6 dorsoventral). To test the stability of aberrant newborn neurons, rats received retrovirus injection 1 week after KA-induced SE and were killed $14 \mathrm{~d}$ [ $\operatorname{control}$ (con), $n=4$; KA, $n=4$ ], 4 weeks (con, $n=$ 5 ; KA, $n=5$ ), 3 months (con, $n=4$; KA, $n=4)$, or 1 year (con, $n=3$; KA, $n=5$ ) thereafter. To analyze the time during which neurogenesis is altered after SE, rats that had seizures were injected 2 months after KA injection with CAG-GFP retrovirus and killed 4 weeks later $(n=5)$. To analyze the effects on newborn cells generated before the onset of seizures, rats were injected with CAG-GFP and seizures were induced 1 week $(n=4)$ or 4 weeks later $(n=3)$. To rule out gender-specific effects on the morphology of neurons born after SE, we injected a group of male Fisher-344 rats ( $8-10$ weeks old; $180-220 \mathrm{~g})$ with CAG-GFP retrovirus 1 week after KA injection and killed the rats 4 weeks later (con, $n=5$; KA, $n=3$ ). The responsiveness of aberrant granule cells was supposed to be tested by challenging rats that were injected with CAG-GFP 1 week after KA-induced SE rats in a novel environment (Tashiro et al., 2007). Two of three rats that had seizures (female; Fisher-344) developed brief episodes of handling-induced seizures (lasting $<5 \mathrm{~min}$ ), were killed $3 \mathrm{~h}$ later, and subsequently used for c-fos analyses. As controls for this experiment, we used rats that had also experienced SE and were injected with CAG-GFP, but had been kept in their standard cages $(n=3)$.

Retrovirus. We used a Moloney murine leukemia retrovirus in which the expression of enhanced GFP is driven by the compound promoter CAG, which contains the cytomegalovirus enhancer chicken $\beta$-actin promoter and a large synthetic intron (CAG-GFP). Retroviruses were produced as described previously (Zhao et al., 2006). Titers ranged between 2.5 and $5 \times 10^{7} \mathrm{cfu} / \mathrm{ml}$.

Immunohistochemistry and light microscopy. Rats were anesthetized with a ketamine/xylazine mixture and transcardially perfused with $0.9 \%$ $\mathrm{NaCl}$ solution followed by $4 \%$ paraformaldehyde (PFA) in $0.1 \mathrm{M}$ phosphate buffer, $\mathrm{pH}$ 7.4. Brains were postfixed overnight in 4\% PFA and then transferred into $30 \%$ sucrose solution. Forty micrometer coronal section series were cut using a sliding microtome (Leica, Nussloch, Germany). Immunostaining was essentially done as described previously (Jessberger and Kempermann, 2003). Primary antibodies used were rabbit $\alpha$-GFP (1:250; Invitrogen, Eugene, OR), chicken $\alpha$-GFP (1:500; Aves, Tigard, OR), rabbit $\alpha$-c-fos (1:200; Santa Cruz Biotechnology, Santa Cruz, CA), mouse $\alpha$-NeuN (1:200; Millipore, Temecula, CA), rabbit $\alpha$-synapsin (1:500; Calbiochem, La Jolla, CA), rabbit $\alpha$-Prox1 (1:5000; Millipore), goat $\alpha$-doublecortin (DCX) (1:250; Santa Cruz), and mouse $\alpha$-calbindin (1:500; Swant, Bellinzona, Switzerland). Secondary antibodies were all obtained from the Jackson Immunoresearch (Bar Harbor, $\mathrm{ME}$ ). Fluorescent signals were detected using a spectral confocal microscope (Radiance 2100; Bio-rad, Hercules, CA). All analyses were performed in sequential scanning mode and double labeling was confirmed by three-dimensional reconstructions of $z$ series. The total number of DCX-positive cells was counted using every 12 th section $(480 \mu \mathrm{m}$ apart $)$ from all animals in the time-course experiment. DCX-expressing cells were counted using a modified version of the optical dissector method where cells in the uppermost focal plane were not included throughout the rostrocaudal extent of the granule cell layer (GCL) using a $20 \times \mathrm{ob}$ jective and an upright epifluorescence microscope (E800; Nikon, Tokyo, Japan). Derived numbers were multiplied by 12 to obtain total cell numbers per GCL. Dendritic architecture was classified as aberrant if there was a dendritic process with abundant spines pointing toward the hilus. Because of sectioning, the number of basal dendrites so classified might be systematically underestimated. Granule cells were considered ectopic when they were located more than two cell layers (approximating $25 \mu \mathrm{m}$ ) away from the inner border of the GCL. Axonal processes were identified by the smaller diameter and lack of dendritic spines compared with dendritic processes extending from newborn granule cells. We analyzed a total of 179 labeled granule cells at the 4 week time point, 134 labeled granule cells at the 3 month time points (virus injection 1 week after KA), and 183 labeled granule cells at the 4 week time point when the virus was injected 2 months after KA injection.

Spine analyses. Spine analyses were performed as described previously (Zhao et al., 2006). Dendritic segments of $\sim 50 \mu \mathrm{m}$ in length were analyzed 4 weeks and 3 months after viral injection in controls and animals that had seizures. At least 16 dendritic segments were analyzed per condition and time point. For apical dendrites, the segments of the distal third of the dendritic tree were imaged. For basal dendrites, only dendrites that extended from somata within the subgranular zone (SGZ) or GCL were selected for imaging, and segments throughout the basal dendritic tree were imaged. All spine analyses were performed using the endogenous GFP signal.

Electron microscopy. SE was induced in two rats, followed 1 week later by retroviral injection. Four weeks after the viral injection, rats were perfused with $4 \%$ paraformaldehyde plus $0.2 \%$ glutaraldehyde and prepared for electron microscopy (EM) as described previously (Toni et al., 2007). Briefly, slices were cut at a thickness of $100 \mu \mathrm{m}$. One to two retrovirally labeled cells per rat were injected under a fluorescence microscope with 5\% aqueous Lucifer yellow (Sigma). Slices were then in-

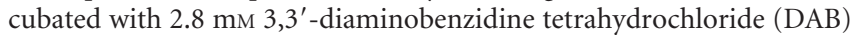
and $6 \mathrm{~mm}$ potassium cyanide and irradiated under conventional epifluorescence using a $75 \mathrm{~W} \mathrm{Hg}$ to induce photoconversion of DAB into an electron-dense residue. Slices were then postfixed overnight in a solution of 3\% glutaraldehyde and processed conventionally for electron microscopy. For serial section EM, 60 serial sections were cut at a thickness of 40 nm. Labeled dendritic segments were cut longitudinally and imaged with a Megaview III camera mounted on a JEOL (Peabody, MA) 100CXII electron microscope at a 19,000 $\times$ magnification. Images were contrasted and stitched using Adobe (San Jose, CA) Photoshop, and aligned and reconstructed using the Align software (provided by J. Fiala, Boston University, Boston, MA).

\section{Results}

Long-lasting aberration of adult neurogenesis after seizures A retroviral strategy was used to label dividing progenitors and their progeny after the induction of SE using KA. One week after KA-induced seizures, we injected a retrovirus encoding GFP into the dentate gyrus of young, adult rats. This time period is when proliferation of neural progenitors in the SGZ of female rats is massively elevated (Jessberger et al., 2007). The animals were killed at several time points thereafter. Four weeks after viral injection, the dendrites of newborn granule cells in control animals showed a highly polarized morphology, and apical dendritic processes with abundant spines extending into the molecular layer (ML), suggesting synaptic integration into the dentate circuitry (Fig. 1A) (van Praag et al., 2002). At this time the axon has also reached its target area, CA3, via the mossy fiber pathway (Zhao et al., 2006). In contrast, KA-induced SE led to dramatic changes in neuronal morphology. In addition to the apical dendritic processes, a population of granule cells born after SE extended additional dendrites deep into the hilus. The aberrant dendrites were covered with spines, suggesting their synaptic connection to hilar structures (Fig. $1 B$ ). Strikingly, many basal dendrites showed a similar branching pattern as apical dendrites on control cells. Basal dendrites commonly ramified their main dendritic processes in the SGZ (Fig. $1 C, D$ ), similar to what was observed for control cells in the distal GCL. The extent of aberrant dendritic growth showed a certain degree of variability, with some areas consisting exclusively of aberrant newborn granule cells (Fig. 1C). The percentages of virus-transduced newborn granule cells with aberrant dendritic morphology were $20.2 \pm$ $3.8 \%$ at 4 weeks and $21.8 \pm 3.1 \%$ at 3 months after virus injection $(p>0.05)$. Despite these dramatic changes in neuronal architecture, neurons born after SE in the dentate area colabeled with the granule cell marker, Prox-1 (data not shown). A number of newborn granule cells ectopically migrated into the hilus (supple- 
mental Fig. 1, available at www.jneurosci.org as supplemental material), where they extended spinous dendrites (Fig. 1E). Approximately, $13.8 \pm 3.8 \%$ and $8.8 \pm$ $1.6 \%$ of virus-transduced newborn neurons were found in the hilus at 4 weeks and 3 months after virus injection, respectively $(p>0.05)$. The formation of spines in the hilus from these ectopic cells and from basal dendrites suggested the synaptic integration of aberrant granule cells in a place where, under normal conditions, a granule cell would never form postsynaptic contacts. In addition, whereas most newborn granule cells in control animals remained in the inner third of the GCL (Fig. 1A) (Kempermann et al., 2003), a significant number of cells migrated into the outer third of the GCL in response to seizure activity (Fig. $1 B$ ).

To determine whether seizure activities exert similar influences on adult neurogenesis in male rats, we analyzed the morphology of 4-week-old neurons in a group of male rats that were born 1 week after KA-induced SE. Indeed, we found both types of aberrant cells: specifically, cells with spiny basal dendrites and ectopic cells in the hilus (supplemental Fig. 2, available at www. jneurosci.org as supplemental material).

Once adult-born granule cells survived an initial critical period, they became stably integrated under normal conditions (Fig. 1F) (Kempermann et al., 2003). We next sought to determine whether aberrantly polarized granule cells survived and thus shaped the dentate output even long after the epileptic insult. Strikingly, a substantial fraction of granule cells extending basal dendrites was found even 1 year after virus injection, indicating the long-term survival of aberrant granule cells born after SE (Fig. 1G).

\section{Distinct effects on granule cells born before or after seizures}

These results unambiguously showed the morphological effects on granule cells that were born shortly after SE. We next asked whether granule cell development continued to be disturbed in the course of the epileptic disease process. To this end, newborn cells were retrovirally labeled 2 months after KA injection and were analyzed 4 weeks later. The polarity of virus-transduced neurons born after SE remained significantly altered even 2 months after the initial insult, when $21.4 \pm 1.4 \%$ of newborn neurons extended spiny basal dendrites toward the hilus (Fig. $2 A)$. The number of newborn granule cells extending basal dendrites was similar to the number of newborn granule cells that we found when we retrovirally labeled the cells 1 week after KA injection $(p>0.05)$. This finding indicates that seizure-induced neurogenesis remains aberrant in the chronic phase after SE, even though DCX numbers return to baseline levels (Fig. $1 H$ ) or decline even further (Hattiangady et al., 2004).

Surprisingly, we found no significant morphological alterations when we injected the retrovirus 1 week before the onset of
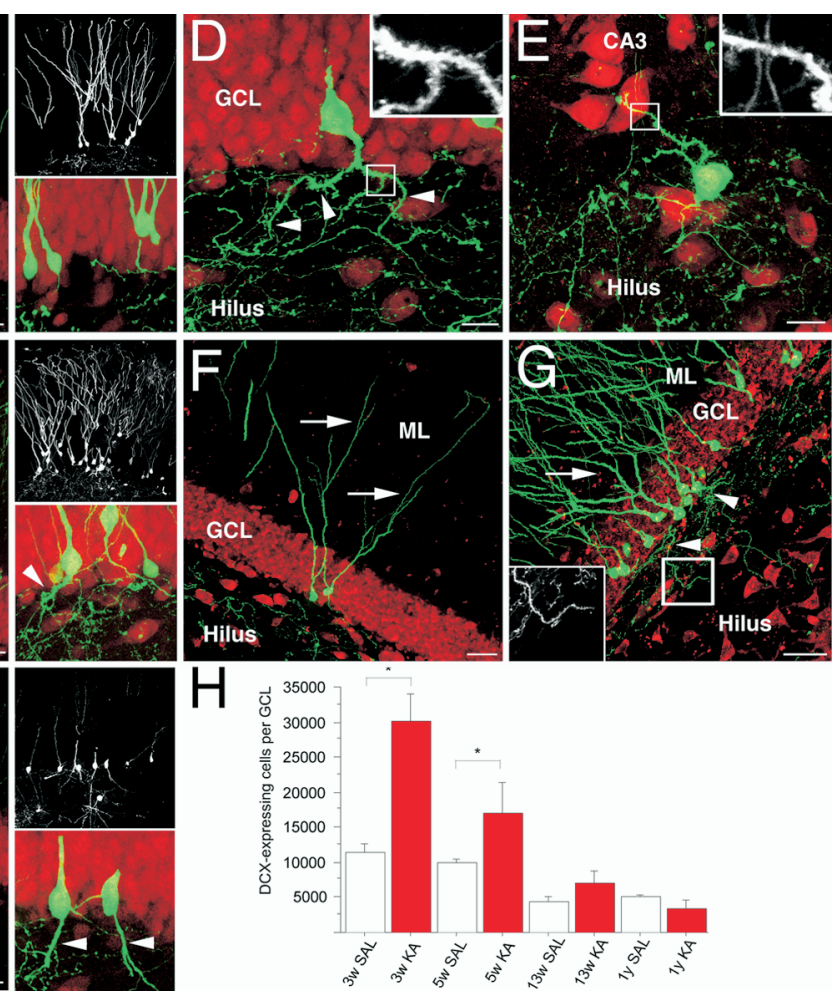

Figure 1. Retroviral labeling of newborn cells in the adult hippocampus after seizure activity. $\boldsymbol{A}-\boldsymbol{G}$, Retroviral labeling of newborn granule cells in controls $(\boldsymbol{A}, \boldsymbol{F})$ and animals that had seizures $(\boldsymbol{B}-\boldsymbol{E}, \boldsymbol{G})$ revealed altered polarity and ectopic localization ule neurons born after $S E$. Whereas control cells 4 weeks after viral injection $(A)$ extended an apical dendrite toward the $M L$, with dendritic spines $(\boldsymbol{D})$. Granule cells born after SE also ectopically migrated into the hilar/CA3 border $(\boldsymbol{E})$, which was never

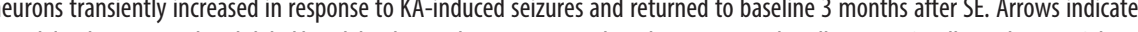

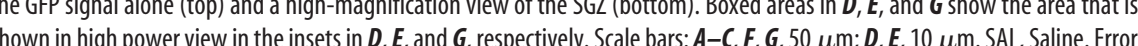
bars in $\boldsymbol{H}$ represent SEM. ${ }^{*} p<0.05$.

SE (Fig. 2B). Similar to control conditions, granule cells born 1 week before KA injection showed regular dendritic polarization and morphology, indicating a critical period for the development of aberrant dendritic processes. We also found no changes in dendritic morphology when we labeled newborn granule cells 4 weeks before SE (Fig. 2B,C). However, a population of those neurons that were relatively mature at the onset of seizures showed mossy fiber sprouting into the proximal ML (Fig. 2D). In contrast, we found no mossy fiber sprouting into the inner ML from newborn granule cells that were born at any time point after the onset of SE (see above).

\section{Seizure activity affects spine maturation}

Given the finding that dendritic growth was perturbed by seizure activity, we next asked whether spine formation or spine maturation of granule neurons born after seizure activity was affected. Fourteen days after viral injection, neither control nor seizureinduced granule cells showed any sign of spiny protrusions on their dendrites (data not shown). The density of spines covering distal segments of the apical dendrites was not different between controls and animals that had seizures 4 weeks and 3 months after virus injection (Fig. $3 A-E$ ). However, the formation of mushroom spines was enhanced in animals that had seizures 3 months 

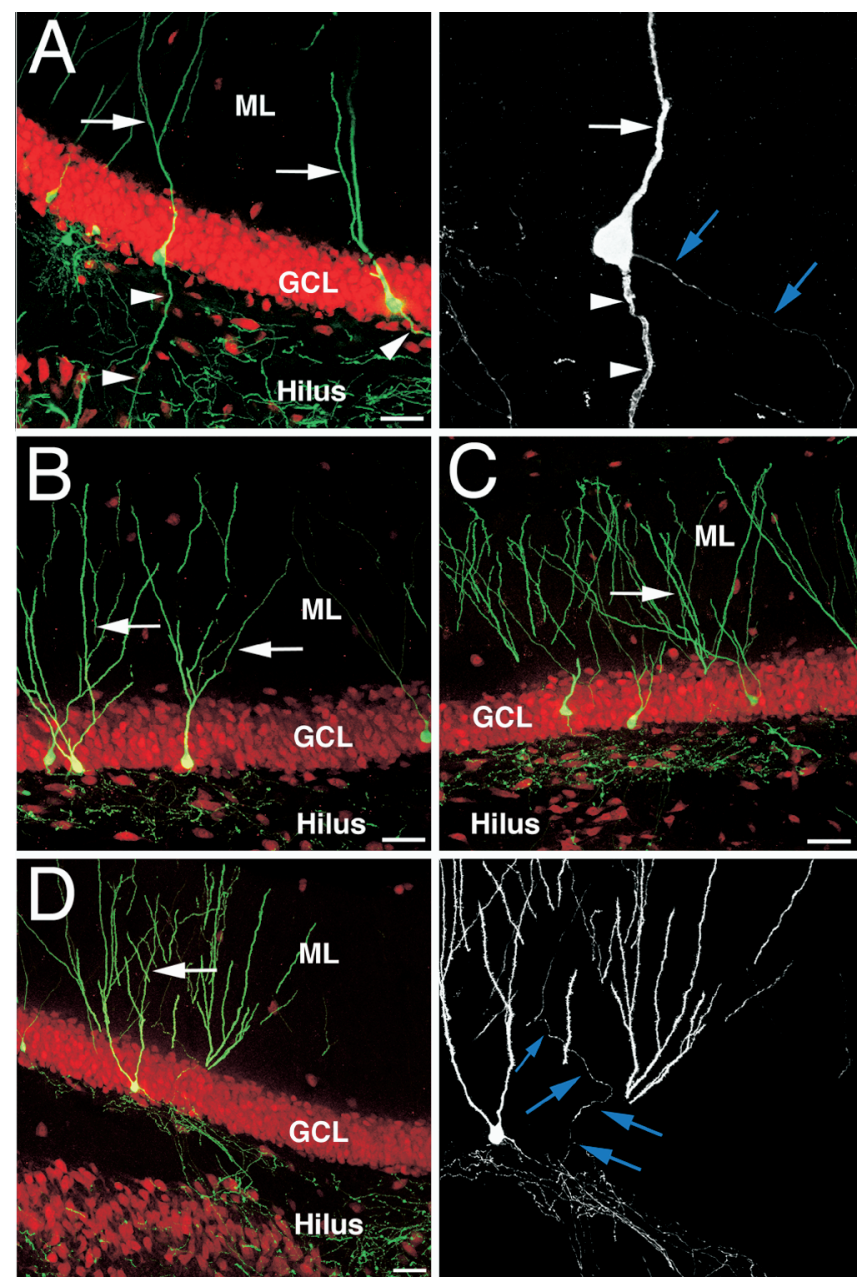

Figure 2. Differential effects on granule cells born before or after onset of SE. $A$, Retroviral labeling of newborn granule cells 2 months after KA injection demonstrated that the detrimental effects of the initial SE on adult neurogenesis are long lasting as, even at this late time point after SE, a substantial fraction of granule cells extended spiny hilar basal dendrites (right panel shows GFP signal only). $\boldsymbol{B}, \boldsymbol{C}$, In contrast, the polarity of newborn granule cells was not significantly altered when dividing progenitors and their progeny were retrovirally labeled 1 week before $S E(B)$, which was also true for the dendritic architecture of new granule cells labeled 4 weeks before KA injection (C). D. However, a substantial fraction of the latter cells showed extensive mossy fiber sprouting into the ML (right panel shows GFP signal only). Arrows indicate apical dendrites; arrowheads label basal dendrites. Blue arrows point toward axonal processes. The pan-neuronal marker NeuN is red in all images. Scale bars, $50 \mu \mathrm{m}$.

after SE compared with controls (Fig. 3E) $(p<0.01)$. We also analyzed basal dendrites from 4 -week-old neurons that were labeled 2 months after the initial insult. Interestingly, the spine density on hilar basal dendrites was similar to that on apical dendrites ( $1.54 \pm 0.097$ per micrometer), with a relatively high number of large mushroom spines $(0.19 \pm 0.026$ per micrometer) compared with distal apical dendrites $(0.12 \pm 0.026$ per micrometer) from control neurons of the same experiment.

In contrast to female rats, we found that spine density was affected by KA-induced seizure in male rats. Spine density of apical dendrites decreased 4 weeks after KA-induced SE (KA, $1.05 \pm 0.074$ per micrometer; control, $1.35 \pm 0.066$ per micrometer; $p<0.01)$. Spine density of basal dendrites in male rats was relatively high $(1.73 \pm 0.0014$ per micrometer $)$ compared with apical dendrites of neurons in either male KA or control groups. Consistent with experiments using female rats, mushroom spine density of apical dendrites was not affected at 4 weeks after seizure
(KA, $0.06 \pm 0.014$ per micrometer; control, $0.04 \pm 0.011$ per micrometer; $p=0.38$ ) in male rats. Basal dendrites of 4 -week-old neurons showed a relatively high number of mushroom spines $(0.17 \pm 0.025$ per micrometer $)$ compared with apical dendrites $(0.04 \pm 0.0011$ per micrometer) extending from granule cells born under control conditions in male rats.

\section{Ectopic integration of aberrant granule cells}

The formation of spines strongly suggested the synaptic integration of aberrant granule cells. To confirm this, we colabeled sections from controls and animals that had seizures with the presynaptic marker protein, synapsin. Dendritic spines in the ML of control granule cells were commonly juxtaposed to synapsin punctae (Fig. 4A). The same was true for spines on basal dendrites arising from granule cells born after SE (Fig. 4B). Using serial section EM, we found that spines from hilar dendrites formed bona fide synapses, as defined by the presence of the following on at least one section: a clear postsynaptic density, at least four presynaptic vesicles within $100 \mathrm{~nm}$ of the presynaptic membrane, and a well defined synaptic cleft. Thus, EM analyses confirmed the aberrant synaptic integration of granule cells born after SE into the dentate area (Fig. $4 C-F$ ).

Functional responsiveness of aberrant granule cells

Although synaptic maturation and normal survival rates of aberrant granule cells might indicate the participation of aberrant granule cells in hippocampal function, we sought additional evidence that these cells become functionally integrated into the dentate gyrus circuitry. The immediate early gene c-fos is dynamically regulated with synaptic activity (Morgan et al., 1987; Gass et al., 1993). Detecting c-fos in newborn neurons after synaptic activation has been used to study the responsiveness of newborn neurons in the adult hippocampus (Jessberger and Kempermann, 2003; Kee et al., 2007; Tashiro et al., 2007). We found expression of c-fos in virus-labeled granule cells extending basal dendrites $3 \mathrm{~h}$ after a single episode of handling-induced seizures (Fig. 5). c-fos expression was also detectable in ectopic, newborn granule cells in the hilus (data not shown), as had been suggested previously by calbindin and c-fos colabeling (Scharfman et al., 2002).

\section{Discussion}

In this study we retrovirally labeled adult-generated granule cells born in male and female adult rats before or after SE induced by KA. A substantial fraction of granule cells born after SE extended abnormal, hilar basal dendrites, but became morphologically and functionally integrated into the dentate circuitry. The effects of SE on hippocampal neurogenesis were long lasting, because even cells born 2 months after the initial SE extended spine-covered basal dendrites deep into the hilus. In contrast, adult-born granule cells that were born before SE did not show aberrant dendritic growth, indicating a maturation-specific effect of SE on newborn granule cells. Thus, our study provides a detailed, retrovirusbased characterization of the effects of SE on dendritic polarization, spine maturation, and integration of granule cells born before or after SE.

Seizure activity in humans leads to a variety of structural changes both outside and within the hippocampal formation (Theodore and Gaillard, 2002). The mechanisms that regulate the progression from single seizures toward epilepsy are still unclear, and why epilepsy is commonly associated with cognitive impairment remains poorly understood (Dalby and Mody, 2001; Elger et al., 2004; Magloczky and Freund, 2005). The finding that 
progenitor cell proliferation and subsequent net neurogenesis are dramatically upregulated in rodent models of mTLE opened up a completely new level of complexity in understanding the pathogenesis and consequences of epilepsy (Parent, 2002).

Previous reports showed that alterations in adult neurogenesis after seizures occurred at the progenitor cell level (Huttmann et al., 2003; Jessberger et al., 2005). There is also emerging evidence that seizure-induced granule cells show abnormal morphological features that are not seen under normal conditions, such as basal dendrites extending into the hilar region of the dentate area (Dashtipour et al., 2003; Shapiro and Ribak, 2006). Importantly, previous studies only used transiently expressed markers like DCX or proopiomelanocortin (POMC)-driven GFP (see below) to identify newborn granule cells (Shapiro et al., 2005; Overstreet-
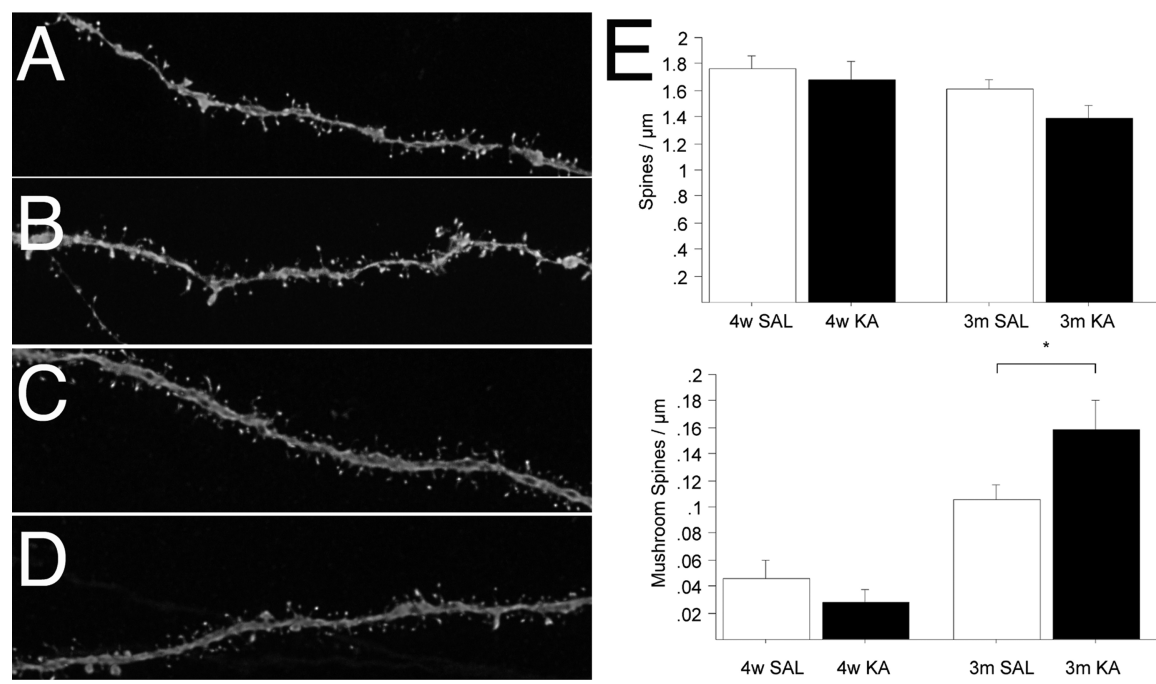

Figure 3. Dendritic spine morphology of granule cells born after SE. $A-E$, Analyses of spine density and shape 4 weeks $(A, B)$ and 3 months $(\boldsymbol{C}, \boldsymbol{D})$ after retrovirus injection in controls $(\boldsymbol{A}, \boldsymbol{C})$ and animals that had seizures $(\boldsymbol{B}, \boldsymbol{D})$ demonstrated that, despite no differences in spine density of apical dendrites, the number of mushroom spines was increased in animals that had seizures compared with controls 3 months after retrovirus injection (E). SAL, Saline. Error bars in $\boldsymbol{E}$ represent SEM. ${ }^{*} p<0.01$.

Wadiche et al., 2006), making it impossi-

ble to analyze the temporal stability of seizure-associated changes regarding granule-cell morphology and the resulting alterations in dentate connectivity. The use of retroviral labeling in this study overcame that problem and allowed, for the first time, the longterm observation of cohorts of granule cells born in response to or before seizures. We show here that aberrant dendrites commonly arise from granule cells born after SE, extend dendritic spines, and synaptically integrate into the pre-existing circuitry. Thus, neurons born after SE have a distinct connectivity compared with control cells, resulting in long-lasting alterations in the dentate circuitry. Furthermore, even granule cells that were born 2 months after the initial insult extended aberrant dendrites toward the hilus. It remains unclear whether the long-lasting effects on neuronal maturation in KA-injected rats are dependent or independent of spontaneous, recurrent seizures that developed in the seizure model used in this study. The intermittent use of anticonvulsants might help to understand the role of ongoing seizures on the alterations of newborn granule-cell morphology long after SE. In any case, seizure-induced neurogenesis clearly has dramatic and long-lasting effects on the structure and connectivity of the hippocampal circuitry. Basal dendrites arising from aberrant granule neurons might receive aberrant polysynaptic input by mossy fiber connections resulting in heightened excitability and disturbance of information processing through the dentate circuitry (Overstreet-Wadiche et al., 2006). Emerging evidence suggests that, under normal conditions, newborn granule cells have distinct physiological properties that might be critically involved in the function of newborn neurons (Wang et al., 2000; Schmidt-Hieber et al., 2004). The aberrant dendrites from granule cells born after SE causing abnormal synaptic connections might affect those unique characteristics of newborn granule cells, even though aberrant granule cells matured into activity-dependent responsiveness as determined by c-fos expression. Surprisingly, a previous study by Jakubs et al. (2006) found that granule cells born after electrical induction of SE did not have any abnormal morphological features compared with new granule cells that were born in running rats. In that study, Jakubs et al. (2006) found increased inhibitory and reduced excitatory input onto granule cells born after SE compared with granule cells generated in the running animals. These results led them to speculate that seizure-induced neurogenesis may mitigate pathological excitation rather than being part of the pathology. Why we and others (Parent et al., 1997; Scharfman et al., 2000; OverstreetWadiche et al., 2006; Shapiro and Ribak, 2006), in contrast to Jakubs et al. (2006), have found dramatic differences in the neuronal morphology of newborn granule cells remains unclear, but it might be explained by the seizure model and/or retroviral vectors used (and subsequent GFP expression levels). Furthermore, the age of granule cells and the time point they were born after SE might be an important factor in regulating the electrophysiological excitability of newborn cells.

In addition to the aberrant dendritic growth of neurons born after KA injection, we found retrovirus-labeled ectopic granule cells in the hilar/CA3 border, as had been described previously using post hoc BrdU detection and granule cell marker expression (Scharfman et al., 2000). Notably, these ectopic granule cells appear to be synchronized with CA3 pyramidal cells and thus might also contribute to heightened excitability after seizures (Scharfman et al., 2000). Supporting an epileptogenic role for neurons born after SE, a previous study showed that the ablation of new neurons generated in response to seizures reduced the occurrence and duration of spontaneous recurrent seizure activity (Jung et al., 2004). Furthermore, seizure-associated cognitive impairment was reduced when seizure-induced neurogenesis was inhibited with the histone deacetylase-inhibitor valproic acid (Jessberger et al., 2007). However, the question of how abnormal connectivity contributes to epileptogenesis or hippocampal dysfunction after seizures can only be answered by analyses of hippocampal physiology with and without the participation of aberrant, newborn granule cells.

There is emerging evidence that the maturation and integration of newborn neurons progress as distinct developmental steps that are at least partially regulated by activity-dependent mechanisms (Ming and Song, 2005; Overstreet Wadiche et al., 2005; Ge et al., 2006; Tashiro et al., 2006). Using transgenic reporter mice expressing GFP under the POMC promoter, a previous study showed that seizure activity accelerates the synaptic integration of newborn granule cells in the adult hippocampus (Overstreet- 

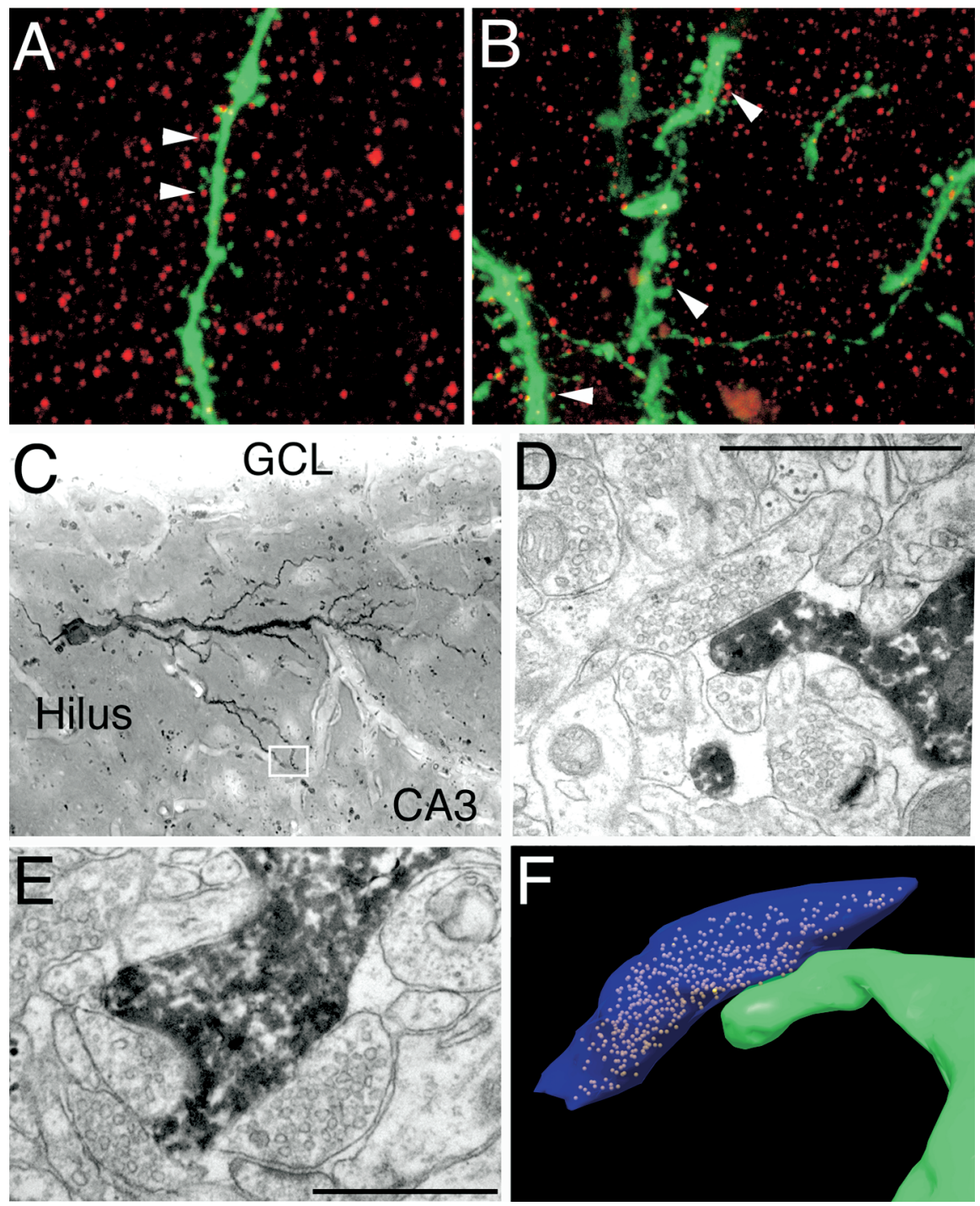

Figure 4. Synaptic integration of aberrant granule cells. $A, B$, Similar to apical dendritic spines of controls $(A)$, spiny processes on basal dendrites extending from neurons born after $S E(B)$ were often in close proximity (arrowheads) to the presynaptic protein synapsin (red). $\boldsymbol{C}-\boldsymbol{E}$, Electron microscopy of aberrant neurons $(\boldsymbol{C})$ confirmed the formation of synapses on ectopic dendrites within the hilar region $(\boldsymbol{D}, \boldsymbol{E}) . \boldsymbol{F}$, Three-dimensional reconstruction of the ectopic dendritic spine (green, shown in $\boldsymbol{D}$ ) contacting an axon (blue) filled with synaptic vesicles. The synapses shown in $\boldsymbol{D}-\boldsymbol{F}$ were located in the area boxed in $\boldsymbol{C}$. Scale bars: $\boldsymbol{D}, \boldsymbol{E}, 1 \mu \mathrm{m}$.

Wadiche et al., 2006). However, this approach only allowed a snapshot observation of newborn cells. We demonstrate here that granule cells born after SE had relatively more mushroom spines on apical dendrites compared with control cells at 3 months after virus injection. We found a similar or higher spine density on aberrant basal dendrites extending from granule cells born after SE compared with apical dendrites. Interestingly, hilar basal dendrites showed a substantial number of spines that had matured into large mushroom spines in both male and female rats at 4 weeks after virus injection, suggesting that these hilar basal dendrites might be more readily influenced by KA-induced seizure activities. In general, the size of the spine head positively correlates with the amount of AMPA-receptors and also influences $\mathrm{Ca}^{2+}$ dynamics (Matsuzaki et al., 2001), thus possibly indicating the strength of the synapse (Hayashi and Majewska, 2005). The enlargement of the spine head has also been associated with LTP (Matsuzaki et al., 2004). However, previous studies showed that not only spine size but also spine neck geometry are critical parameters regulating the size of the electrical potential at the neu- ronal soma (Noguchi et al., 2005; Araya et al., 2006). How the relative change in spine geometry after seizures translates into function remains unknown. One possibility is that synapses onto spines of hilar basal dendrites might be more active than synapses onto spines of apical dendrites, leading to the formation of more mushroom spines on basal dendrites. Future studies will be needed to systematically examine the electrophysiological properties of aberrant neurons generated in response to seizures.

It is unlikely, that epilepsy and cognitive impairment in the course of the disease have a single cause, but rather are associated with a variety of structural and molecular changes. We show here that seizures have different effects on granule-cell maturation and morphology depending on the age of the newborn cell in relation to the insult. Clearly, there is a critical period during which dendritic pathfinding is vulnerable to external stimuli. However, the vulnerable phase seems to be different for sprouting of axonal processes into the ML, which we exclusively observed in newborn neurons that were at least 4 weeks old. This finding is consistent with previous observations that ablation of neurogenesis does not prevent mossyfiber sprouting into the ML after seizures (Parent et al., 1999). The molecular mechanisms underlying the distinct vulnerability of dendritic versus axonal pathfinding remain unknown. The results presented here define the critical age of newborn granule cells in relation to initial seizures in which either aberrant dendritic growth or axonal sprouting is likely to occur.

The reasons for seizure-induced alteration of hippocampal neurogenesis probably lie both in cell-intrinsic alterations of progenitors and/or immature neurons and in environmental changes, such as glial activation, and secretion of neurotrophic factors. In addition, excitotoxic cell death occurring in the seizure model used here (Jessberger et al., 2007) might be critically associated with alterations of adult neurogenesis after SE. Interestingly, our data also suggest that gender appears to influence the formation and maturation of spines after SE.

Whether aberrant dendritic growth is caused by changes in intrinsic progenitor/immature neuron properties or environmental cues is not clear. Accumulating evidence supports the idea that the integration of a newborn neuron into the preexisting circuitry relies on a balance between extrinsic signaling and intrinsic cellular properties (Abrous et al., 2005; Ming and Song, 2005). KA-induced seizure activity apparently impairs this balance and leads to the generation of aberrant newborn granule cells.

Our study provides a fundamental framework for identifying the underlying molecular mechanisms of seizure-induced neuro- 
genesis and for characterizing the functional consequences of aberrant neurogenesis in the epileptic disease process.

\section{References}

Abrous DN, Koehl M, Le Moal M (2005) Adult neurogenesis: from precursors to network and physiology. Physiol Rev 85:523-569.

Araya R, Jiang J, Eisenthal KB, Yuste R (2006) The spine neck filters membrane potentials. Proc Natl Acad Sci USA 103:17961-17966.

Bengzon J, Kokaia Z, Elmér E, Nanobashvili A, Kokaia M, Lindvall O (1997) Apoptosis and proliferation of dentate gyrus neurons after single and intermittent limbic seizures. Proc Natl Acad Sci USA 94:10432-10437.

Dalby NO, Mody I (2001) The process of epileptogenesis: a pathophysiological approach. Curr Opin Neurol 14:187-192.

Dashtipour K, Wong AM, Obenaus A, Spigelman I, Ribak CE (2003) Temporal profile of hilar basal dendrite formation on dentate granule cells after status epilepticus. Epilepsy Res 54:141-151.

Elger CE, Helmstaedter C, Kurthen M (2004) Chronic epilepsy and cognition. Lancet Neurol 3:663-672.

Gass P, Herdegen T, Bravo R, Kiessling M (1993) Spatiotemporal induction of immediate early genes in the rat brain after limbic seizures: effects of NMDA receptor antagonist MK-801. Eur J Neurosci 5:933-943.

Ge S, Goh EL, Sailor KA, Kitabatake Y, Ming GL, Song H (2006) GABA regulates synaptic integration of newly generated neurons in the adult brain. Nature 439:589-593.

Hattiangady B, Rao MS, Shetty AK (2004) Chronic temporal lobe epilepsy is associated with severely declined dentate neurogenesis in the adult hippocampus. Neurobiol Dis 17:473-490.

Hayashi Y, Majewska AK (2005) Dendritic spine geometry: functional implication and regulation. Neuron 46:529-532.

Helmstaedter C (2002) Effects of chronic epilepsy on declarative memory systems. Prog Brain Res 135:439-453.

Helmstaedter C, Kurthen M, Lux S, Reuber M, Elger CE (2003) Chronic epilepsy and cognition: a longitudinal study in temporal lobe epilepsy. Ann Neurol 54:425-432.

Huttmann K, Sadgrove M, Wallraff A, Hinterkeuser S, Kirchhoff F, Steinhauser C, Gray WP (2003) Seizures preferentially stimulate proliferation of radial glia-like astrocytes in the adult dentate gyrus: functional and immunocytochemical analysis. Eur J Neurosci 18:2769-2778.

Jakubs K, Nanobashvili A, Bonde S, Ekdahl CT, Kokaia Z, Kokaia M, Lindvall O (2006) Environment matters: synaptic properties of neurons born in the epileptic adult brain develop to reduce excitability. Neuron 52:1047-1059.

Jessberger S, Kempermann G (2003) Adult-born hippocampal neurons mature into activity-dependent responsiveness. Eur J Neurosci 18:2707-2712.

Jessberger S, Romer B, Babu H, Kempermann G (2005) Seizures induce proliferation and dispersion of doublecortin-positive hippocampal progenitor cells. Exp Neurol 196:342-351.

Jessberger S, Nakashima K, Clemenson Jr GD, Mejia E, Mathews E, Ure K, Ogawa S, Sinton CM, Gage FH, Hsieh J (2007) Epigenetic modulation of seizure-induced neurogenesis and cognitive decline. J Neurosci 27:5967-5975.

Jung KH, Chu K, Kim M, Jeong SW, Song YM, Lee ST, Kim JY, Lee SK, Roh JK (2004) Continuous cytosine-b-D-arabinofuranoside infusion reduces ectopic granule cells in adult rat hippocampus with attenuation of spontaneous recurrent seizures following pilocarpine-induced status epilepticus. Eur J Neurosci 19:3219-3226.

Kee N, Teixeira CM, Wang AH, Frankland PW (2007) Preferential incorporation of adult-generated granule cells into spatial memory networks in the dentate gyrus. Nat Neurosci 10:355-362.

Kempermann G, Gast D, Kronenberg G, Yamaguchi M, Gage FH (2003) Early determination and long-term persistence of adult-generated new neurons in the hippocampus of mice. Development 130:391-399.
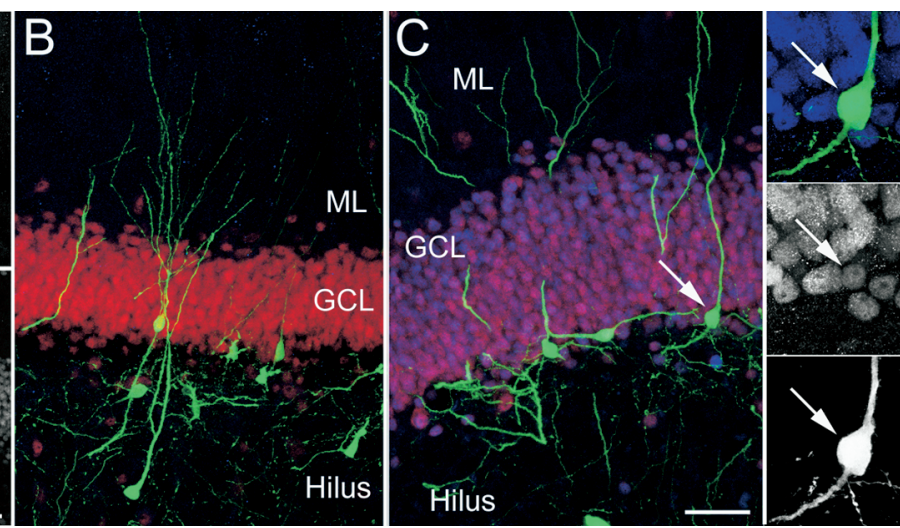

Hilus

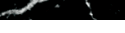

Figure 5. Activity-induced c-fos expression in aberrant granule cells extending basal dendrites. $\boldsymbol{A}$, Three hours after a seizure episode in epileptic rats (SE 5 weeks earlier), c-fos was dramatically upregulated in the dentate gyrus

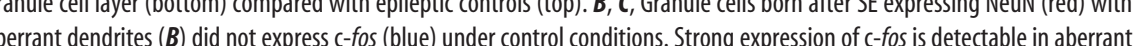
granule cells $(\boldsymbol{C}) 3 \mathrm{~h}$ after handling-induced seizure activity. Insets in $\boldsymbol{C}$ show a high-power view of the newborn granule cell

Magloczky Z, Freund TF (2005) Impaired and repaired inhibitory circuits in the epileptic human hippocampus. Trends Neurosci 28:334-340.

Matsuzaki M, Ellis-Davies GC, Nemoto T, Miyashita Y, Iino M, Kasai H (2001) Dendritic spine geometry is critical for AMPA receptor expression in hippocampal CA1 pyramidal neurons. Nat Neurosci 4:1086-1092.

Matsuzaki M, Honkura N, Ellis-Davies GC, Kasai H (2004) Structural basis of long-term potentiation in single dendritic spines. Nature 429:761-766.

Ming GL, Song H (2005) Adult neurogenesis in the mammalian central nervous system. Annu Rev Neurosci 28:223-250.

Morgan JI, Cohen DR, Hempstead JL, Curran T (1987) Mapping patterns of c-fos expression in the central nervous system after seizure. Science 237:192-197.

Noguchi J, Matsuzaki M, Ellis-Davies GC, Kasai H (2005) Spine-neck geometry determines NMDA receptor-dependent $\mathrm{Ca}^{2+}$ signaling in dendrites. Neuron 46:609-622.

Overstreet Wadiche L, Bromberg DA, Bensen AL, Westbrook GL (2005) GABAergic signaling to newborn neurons in dentate gyrus. J Neurophysiol 94:4528-4532.

Overstreet-Wadiche LS, Bromberg DA, Bensen AL, Westbrook GL (2006) Seizures accelerate functional integration of adult-generated granule cells. J Neurosci 26:4095-4103.

Parent JM (2002) The role of seizure-induced neurogenesis in epileptogenesis and brain repair. Epilepsy Res 50:179-189.

Parent JM, Yu TW, Leibowitz RT, Geschwind DH, Sloviter RS, Lowenstein DH (1997) Dentate granule cell neurogenesis is increased by seizures and contributes to aberrant network reorganization in the adult rat hippocampus. J Neurosci 17:3727-3738.

Parent JM, Tada E, Fike JR, Lowenstein DH (1999) Inhibition of dentate granule cell neurogenesis with brain irradiation does not prevent seizureinduced mossy fiber synaptic reorganization in the rat. J Neurosci 19:4508-4519.

Parent JM, Valentin VV, Lowenstein DH (2002) Prolonged seizures increase proliferating neuroblasts in the adult rat subventricular zoneolfactory bulb pathway. J Neurosci 22:3174-3188.

Racine RJ (1972) Modification of seizure activity by electrical stimulation. II. Motor seizure. Electroencephalogr Clin Neurophysiol 32:281-294.

Scharfman HE, Goodman JH, Sollas AL (2000) Granule-like neurons at the hilar/CA3 border after status epilepticus and their synchrony with area CA3 pyramidal cells: functional implications of seizure-induced neurogenesis. J Neurosci 20:6144-6158.

Scharfman HE, Sollas AL, Goodman JH (2002) Spontaneous recurrent seizures after pilocarpine-induced status epilepticus activate calbindinimmunoreactive hilar cells of the rat dentate gyrus. Neuroscience 111:71-81.

Schmidt-Hieber C, Jonas P, Bischofberger J (2004) Enhanced synaptic plasticity in newly generated granule cells of the adult hippocampus. Nature 429:184-187. 
Shapiro LA, Ribak CE (2006) Newly born dentate granule neurons after pilocarpine-induced epilepsy have hilar basal dendrites with immature synapses. Epilepsy Res 69:53-66.

Shapiro LA, Korn MJ, Ribak CE (2005) Newly generated dentate granule cells from epileptic rats exhibit elongated hilar basal dendrites that align along GFAP-immunolabeled processes. Neuroscience 136:823-831.

Stafstrom CE, Chronopoulos A, Thurber S, Thompson JL, Holmes GL (1993) Age-dependent cognitive and behavioral deficits after kainic acid seizures. Epilepsia 34:420-432.

Stefan H, Pauli E (2002) Progressive cognitive decline in epilepsy: an indication of ongoing plasticity. Prog Brain Res 135:409-417.

Tashiro A, Sandler VM, Toni N, Zhao C, Gage FH (2006) NMDA-receptormediated, cell-specific integration of new neurons in adult dentate gyrus. Nature 442:929-933.

Tashiro A, Makino H, Gage FH (2007) Experience-specific functional modification of the dentate gyrus through adult neurogenesis: a critical period during an immature stage. J Neurosci 27:3252-3259.

Theodore WH, Gaillard WD (2002) Neuroimaging and the progression of epilepsy. Prog Brain Res 135:305-313.

Toni N, Teng EM, Bushong EA, Aimone JB, Zhao C, Consiglio A, van Praag H, Martone ME, Ellisman MH, Gage FH (2007) Synapse formation on neurons born in the adult hippocampus. Nat Neurosci 10:727-734.

van Praag H, Schinder AF, Christie BR, Toni N, Palmer TD, Gage FH (2002) Functional neurogenesis in the adult hippocampus. Nature 415:1030-1034.

von Lehe M, Lutz M, Kral T, Schramm J, Elger CE, Clusmann H (2006) Correlation of health-related quality of life after surgery for mesial temporal lobe epilepsy with two seizure outcome scales. Epilepsy Behav 9:73-82.

Wang S, Scott BW, Wojtowicz JM (2000) Heterogenous properties of dentate granule neurons in the adult rat. J Neurobiol 42:248-257.

Zhao C, Teng EM, Summers Jr. RG, Ming GL, Gage FH (2006) Distinct morphological stages of dentate granule neuron maturation in the adult mouse hippocampus. J Neurosci 26:3-11. 